\title{
APPROXIMATION BY POLYNOMIALS IN $z$ AND ANOTHER FUNCTION ${ }^{1}$
}

\author{
KENNETH JOHN PRESKENIS
}

\begin{abstract}
We present some progress in the understanding of when and why polynomials in $z$ and a given continuous function $f$ are uniformly dense in all continuous complex valued functions on the closed unit disk. The first theorem requires that $f$ be an $\mathrm{ACL}^{2}$-function in a neighborhood of the disk which satisfies $\operatorname{Re} f_{\bar{z}}>\left|f_{z}\right|$ almost everywhere and $f^{-1}(f(a))$ is countable for each $a$. The second theorem requires that $f$ have a special form and satisfy $\left|f_{\bar{z}}\right|>\left|f_{z}\right|$ everywhere except at the origin. The form is that $f=$ $\bar{z}^{k} \phi\left(|z|^{2 k}\right)$ where $\phi$ is a complex valued function of a real variable satisfying $\phi$ is continuous in $[0,1], \phi^{\prime}$ exists in $(0,1)$ and where $k$ is a positive integer.
\end{abstract}

1. Introduction. Denote by $\mathbf{C}$ the complex plane and by $z$ the identity function. $D$ denotes the closed disk $\{s \in \mathbf{C}:|s| \leqslant 1\}, C(D)$ will be the set of all complex valued continuous functions on $D$ and for $f$ in $C(D), P_{f}$ will be the uniform closure on $D$ of all finite sums $\sum_{i, j>0} a_{i j} z^{i} f^{j}$ where $a_{i j} \in \mathbf{C}$. If $f=U+i V$, then the Jacobian determinant of $f$ is given by $\operatorname{det}\left(U_{x} U_{x}\right)$. Alternately, if we put $f_{z}=\left(U_{x}+V_{y}\right) / 2+i\left(V_{x}-U_{y}\right) / 2$ and $f_{\bar{z}}=\left(U_{x}-\right.$ $\left.V_{y}\right) / 2+i\left(V_{x}+U_{y}\right) / 2$, then direct computation yields $U_{x} V_{y}-U_{y} V_{x}=\left|f_{z}\right|^{2}$ $-\left|f_{\bar{z}}\right|^{2}$. In particular, $f$ has negative Jacobian determinant if and only if $\left|f_{\bar{z}}\right|>\left|f_{z}\right|$. Finally, Lebesgue two dimensional measure in $\mathbf{C}$ is denoted by $m$.

We are concerned with the following open question. Assume $f$ is smooth in a neighborhood of $D$ and $\left|f_{\bar{z}}\right|>\left|f_{z}\right|$; is $P_{f}=C(D)$ ? Our main frame of reference consists of two papers ([6] and [7]) of J. Wermer and two papers ([3] and [4]) by the author. In [3], it is shown that $\left|f_{\bar{z}}\right|>\left|f_{z}\right|$ implies all rational functions in $z$ and $f$, which are finite, are uniformly dense in $C(D)$ and in [4] it is proved that if $\operatorname{Re} f_{\bar{z}} \geqslant\left|f_{z}\right|$ everywhere in the interior of $D$, then $P_{f}=$ $C(D)$.

In §2, we prove the following theorem and a mild extension of it.

THEOREM I. Let $f$ be an $A C L^{2}$-function in a neighborhood of $D$ which satisfies

(i) $\operatorname{Re} f_{\bar{z}} \geqslant\left|f_{z}\right|$ a.e. in $D$ and

(ii) $f^{-1}(f(a))$ is countable for each $a$ in $D$.

Then $P_{f}=C(D)$.

In $\$ 3$, we prove the following theorem.

Received by the editors July $18,1977$.

AMS (MOS) subject classifications (1970). Primary 46J10, 56J15; Secondary 30A60, 30A82.

'This work was partially done while the author was at Boston College on a faculty fellowship. 
THEOREM II. Assume $\phi$ is a complex valued function of a real variable satisfying $\phi$ is continuous in $[0,1]$ and $\phi^{\prime}$ exists in $(0,1)$. Put $f=\bar{z}^{k} \phi\left(|z|^{2 k}\right)$ where $k$ is a fixed but arbitrary positive integer. If $\left|f_{\bar{z}}\right|>\left|f_{z}\right|$ everywhere in Int $D-\{0\}$, then $P_{f}=C(D)$.

The author is grateful to Bruce Palka for helpful suggestions concerning the main theorem in \$2, and to Andrew Browder for helpful conversations about $\S \S 2$ and 3.

2. Before we prove Theorem I, we quote some needed definitions and results which may be found in [5].

Let $Q$ be a closed rectangle in C. A complex valued function $f$ on $Q$ is said to be ACL or absolutely continuous on lines if $f$ is continuous in $Q$ and if $f$ is absolutely continuous on almost every line segment in $Q$ parallel to the coordinate axes. A function $f$ on an open set $U$ in $\mathbf{C}$ is ACL when $f$ restricted to any rectangle in $U$ is ACL. An ACL-function on an open set $U$ has partial derivatives a.e. in $U$ and they are Borel functions. When the partial derivatives of an ACL-function $f$ are locally $L^{2}$-integrable, $f$ is said to be $\mathrm{ACL}^{2}$. (Reference: \$26 in [5].)

Definition. Given a family $\Gamma$ of locally rectifiable paths in $\mathbf{C}$, let $F(\Gamma)$ denote all Borel functions $\rho: \mathbf{C} \rightarrow[0, \infty]$ such that $\int_{\gamma} p d s \geqslant 1$ for each $\gamma$ in $\Gamma$. The modulus of $\Gamma$, denoted by $M(\Gamma)$, is the number $\inf _{\rho \in F(\Gamma)} \int_{C} \rho^{2} d m$; if $F(\Gamma)=\varnothing$, then $M(\Gamma)=\infty$.

THEOREM 2.1. $M(\Gamma)$ has the following properties:

(i) $M(\Gamma)$ is invariant under similarity mappings,

(ii) $M(\varnothing)=0$,

(iii) if $\Gamma_{1} \subset \Gamma_{2}$, then $M\left(\Gamma_{1}\right) \leqslant M\left(\Gamma_{2}\right)$,

(iv) $M\left(\cup_{i=1}^{\infty} \Gamma_{i}\right) \leqslant \sum_{i=1}^{\infty} M\left(\Gamma_{i}\right)$,

(v) if each $\gamma$ in $\Gamma_{2}$ has a subcurve which belongs to $\Gamma_{1}$, then $M\left(\Gamma_{2}\right) \leqslant M\left(\Gamma_{1}\right)$.

For a proof, see $\$ 6$ in [5].

Proposition 2.2. Let $0<\varepsilon<R<\infty$ and let $S(\varepsilon), S(R)$ equal respectively the circles about 0 with radii $\varepsilon, R$. Let $Y$ be a measurable set on the unit circle and take $\Gamma=$ all radial line segments $\gamma$ joining $S(\varepsilon)$ to $S(R)$ such that $|\gamma| \subset\{s \in \mathrm{C}: s /|s| \in Y\}$. Then $M(\Gamma)=\sigma(Y)(\log R / \varepsilon)^{-1}$ where $\sigma(Y)=$ $2(m\{r u: 0<r<1$ and $u \in Y\})$.

In order to prove the proposition, one can mildly modify the argument given in Example 7.5 of [5].

Theorem 2.3 (Fuglede). Suppose $U$ is an open set in $\mathbf{C}$ and that $f$ is an $A C L^{2}$-function on $U$. Let $T$ be the family of all locally rectifiable paths in $U$ which have a closed subpath on which $f$ is not absolutely continuous. Then $M(T)=0$.

Proof of Theorem I. It suffices to show that for $s, t$ in $D$ we have $\operatorname{Re}[(t-s)(f(t)-f(s))] \geqslant 0$. That $P_{f}=C(D)$ will follow from this fact and 
from the hypothesis that $f^{-1}(f(a))$ is countable for each $a$ exactly as in Theorem A of [4]. Also, the continuity of $f$ allows us to restrict our attention to the situation $t \in D, s \in \operatorname{Int} D$ and $t \neq s$. Fix such an $s$ and $t$. Choose $\varepsilon>0$ so small that $t \notin D(s ; \varepsilon)=\{w:|w-s| \leqslant \varepsilon\} \subset$ Int $D$ and let $s_{\varepsilon}=s+$ $\varepsilon(t-s) /|t-s|$. Again, by the continuity of $f$, it suffices to prove that $\operatorname{Re}\left[\left(t-s_{\varepsilon}\right)\left(f(t)-f\left(s_{\varepsilon}\right)\right)\right] \geqslant 0$ and this is what we will show.

Choose $T$ as in Theorem 2.3; there are two cases to consider.

Case I. The line segment $\nu$ joining $s_{\varepsilon}$ to $t$ is not in $T$ and $\operatorname{Re} f_{\bar{z}} \geqslant\left|f_{z}\right|$ a.e. with respect to linear measure on $\nu$.

By Theorem 2.3

$$
f(t)-f\left(s_{\varepsilon}\right)=\int_{\nu}\left(f_{x} d x+f_{y} d y\right)
$$

and so

$$
\operatorname{Re}\left[\left(t-s_{\varepsilon}\right)\left(f(t)-f\left(s_{\varepsilon}\right)\right)\right]=\int_{\nu} \operatorname{Re}\left[\left(t-s_{\varepsilon}\right) d f\left(t-s_{\varepsilon}\right)\right] .
$$

A straightforward calculation yields

$$
\operatorname{Re}\left[\left(t-s_{\varepsilon}\right) d f\left(t-s_{\varepsilon}\right)\right]=\operatorname{Re}\left[\left(t-s_{\varepsilon}\right)^{2} f_{z}+\left|t-s_{\varepsilon}\right|^{2} f_{\bar{z}}\right] .
$$

And our assumption that $\operatorname{Re} f_{\bar{z}} \geqslant\left|f_{z}\right|$ implies that the last expression is nonnegative almost everywhere. We conclude that

$$
\operatorname{Re}\left[\left(t-s_{\varepsilon}\right)\left(f(t)-f\left(s_{\varepsilon}\right)\right)\right] \geqslant 0 .
$$

Case II. Either the line segment from $s_{\varepsilon}$ to $t$ is in $T$ or it is not true that $\operatorname{Re} f_{\bar{z}} \geqslant\left|f_{z}\right|$ a.e. on that segment.

Let $X=\left\{w\right.$ in $D: f_{x}(w)$ or $f_{y}(w)$ does not exist or, if they exist, $\operatorname{Re} f_{\bar{z}}(w)<$ $\left.\left|f_{z}(w)\right|\right\}$. For each $\alpha \in[0,2 \pi]$ denote by $L(\alpha)$ the radial (with center $=s$ ) segment joining $s+\varepsilon e^{i \alpha}$ to the unit circle and let $B=\{\alpha \in[0,2 \pi]: X \cap$ $L(\alpha)$ has positive linear measure $\}$.

It is not hard to show that $m_{1} B=0$ where $m_{1}$ is Lebesgue measure on the real line; for instance, Fubini's theorem may be applied to this situation. Next, let $A=\{\alpha \in[0,2 \pi]: L(\alpha) \in T\}$. It will now be shown $A$ has the property that if $G$ is a measurable subset of $A$, then $m_{1} G=0$.

Let $G$ be a measurable subset of $A$ and let $Y=\left\{e^{i \alpha}: \alpha \in G\right\}$. Choose $R>\varepsilon$ so large that $D \subset D(s ; R)$. Put $L=\{L(\alpha): \alpha \in G\}$ and $L^{\prime}=$ all extensions of segments in $L$ to the circle $S(s ; R)$. On the one hand, $M\left(L^{\prime}\right)=\sigma(Y)(\log R / \varepsilon)^{-1}$ by Proposition 2.2 (and Theorem 2.1, part (i)); on the other hand $L \subset T$ and each path in $L^{\prime}$ has a subpath in $L$ so that (again by Theorem 2.1) $M\left(L^{\prime}\right) \leqslant M(L) \leqslant M(T)$. Since $T$ is from Theorem 2.3, $M(T)=0$. Thus, $M\left(L^{\prime}\right)=0$ and $\sigma(Y)=0$; from which it follows that $m_{1} G=0$.

Now since any measurable subset of $A$ has $m_{1}$-measure zero and since $m_{1} B=0$, any measurable subset of $A \cup B$ has $m_{1}$-measure zero. We conclude that $A \cup B$ contains no intervals. Therefore, the line segment from $s_{\varepsilon}$ to $t$ (although it may be in $T$ ) can be approximated arbitrarily close by radial 
segments which are not in $T$. Choose $a_{n}, b_{n}$ on such line segments such that $a_{n} \in S(s ; \varepsilon), a_{n} \rightarrow s_{\varepsilon}, b_{n} \rightarrow t$. By Theorem 2.3, the Case I situation applies to yield $\operatorname{Re}\left[\left(b_{n}-a_{n}\right)\left(f\left(b_{n}\right)-f\left(a_{n}\right)\right)\right] \geqslant 0$. The continuity of $f$ implies $\operatorname{Re}[(t-$ $\left.\left.s_{e}\right)\left(f(t)-f\left(s_{e}\right)\right)\right] \geqslant 0$ and by the comments at the start of the proof, Theorem $\mathrm{I}$ is proved.

Next, we indicate a mild extension of Theorem $\mathrm{I}$.

THEOREM. Suppose $g$ is a function which satisfies the hypothesis of Theorem I and suppose $R$ is a function in $C(D)$ which satisfies $|R(t)-R(s)| \leqslant k \mid g(t)-$ $g(s) \mid$ for all $t, s$ in $D$ with $k$ a positive constant between 0 and 1 . If $f=g+R$, then $P_{f}=C(D)$.

An example of such a function $R$ can be constructed by taking any real valued function of a real variable, say $h$, which satisfies $\left|h^{\prime}\right| \leqslant k<1$ and putting $R=h(\operatorname{Im} g)-i h(\operatorname{Re} g)$. In order to prove this extension, a couple of observations are relevant. First, for each $s \in D, f^{-1}(f(s))=g^{-1}(g(s))$; this is a direct consequence of the Lipschitz condition on $R$. Since $g^{-1}(g(s))$ is countable, $f^{-1}(f(s))$ is also. Next, for each $s$ the function $(z-s)(f-f(s))$ never takes a negative real number for a value in $D$. In fact, for any $\varepsilon>0$ and any $t \in D$

$$
\begin{aligned}
|(t-s)(f(t)-f(s))+\varepsilon| \\
\quad=|(t-s)(g(t)-g(s))+\varepsilon+(t-s)(R(t)-R(s))| \\
\quad \geqslant|(t-s)(g(t)-g(s))+\varepsilon|-|(t-s)(R(t)-R(s))| \\
\quad \geqslant|(t-s)(g(t)-g(s))+\varepsilon|-k|(t-s)(g(t)-g(s))| .
\end{aligned}
$$

And this last expression is positive since $\operatorname{Re}[(t-s)(g(t)-g(s))] \geqslant 0$. In order to finish a proof of the extension, it suffices to combine an argument used by J. Wermer in Lemma 3 of [7] with the argument in Theorem A of [4].

3. In order to have $P_{f}=C(D)$ it is not sufficient to assume that $f_{\bar{z}} \neq 0$ everywhere. This is demonstrated by an example of J. Wermer, namely, $f=\bar{z} \phi\left(|z|^{2}\right)$ where $\phi(t)=\left(t^{2}-1\right) / 3+i(t-1) / 2$. Straight calculation shows $f_{\bar{z}}$ vanishes nowhere in $\mathbf{C}$ and $f$ itself vanishes on the unit circle. Hence, if $g \in P_{f}$, then $g$, restricted to the unit circle, is approximable by polynomials in $z$. It follows that $P_{f} \neq C(D)$.

This partially leads us to consider the class of functions which have the special form $f=\bar{z}^{k} \phi\left(|z|^{2 k}\right)$ where $\phi$ is a complex valued function of a real variable and where $k$ is an arbitrary but fixed positive integer. For instance, some polynomials in $z$ and $\bar{z}$ can be expressed in this form with $\phi$ being a polynomial in a real variable which has complex coefficients.

Before proving Theorem II, we supply additional information which will be needed.

Theorem 3.1 (LAVRentiev). Let $X$ be a compact subset of $\mathrm{C}$ and let $P(X)$ denote the uniform closure on $X$ of all polynomials in $z$. If $X$ has empty interior 
and connected complement, then $P(X)=C(X)$.

A functional-analytic proof of this result may be found in [1].

TheOREM 3.2. Let $X$ be a compact subset of $\mathrm{C}$ and let $A$ be a closed subalgebra of $C(X)$. Let $g$ be a real valued function in $A$ and for each real number $\alpha$ put $X_{\alpha}=g^{-1}(\alpha)$. If $A$ restricted to each $X_{\alpha}$ is dense in $C\left(X_{\alpha}\right)$ for each $\alpha$, then $A=C(X)$.

This is a special case of a theorem due to Silov (and, in greater generality due to Bishop). For a simple proof which was discovered by Glicksberg and which is based on an argument of De Branges in [2], the reader should see Theorem 2.7.5 in [1].

LEMMA 3.3. Let $f$ be as in Theorem II. Then the function $z^{k} f$ maps $D$ onto a Jordan arc $\Gamma$ where distinct circles about 0 are mapped to distinct points of $\Gamma$.

Proof of Lemma 3.3. Straightforward calculation yields

$$
f_{z}=k \bar{z}^{k+1} \phi^{\prime}\left(|z|^{2 k}\right)|z|^{2 k-2}
$$

and

$$
f_{\bar{z}}=k \bar{z}^{k-1} \phi^{\prime}\left(|z|^{2 k}\right)|z|^{2 k}+k \bar{z}^{k-1} \phi\left(|z|^{2 k}\right)
$$

in Int $D$. Hence,

$$
\left|f_{z}\right|^{2}-\left|f_{\bar{z}}\right|^{2}=-k^{2}\left|z^{k-1}\right|^{2}\left(\left|\phi\left(|z|^{2 k}\right)\right|^{2}+2 \operatorname{Re}\left[|z|^{2 k} \phi\left(|z|^{2 k}\right) \overline{\phi^{\prime}\left(|z|^{2 k}\right)}\right]\right) .
$$

Let $t=|z|^{2 k}$ and $H(t)=|\phi(t)|^{2}$. The hypotheses on $f$ imply that $|\phi(t)|^{2}+$ $2 \operatorname{Re}\left[t \phi(t) \overline{\phi^{\prime}(t)}\right]>0$ or that $[t H(t)]^{\prime}>0$ when $0<t<1$. Thus, $t \rightarrow t H(t)$ is a strictly increasing function of $t$. Next, if $0<r<s<1$, then we have $r^{2}|\phi(r)|^{2}<r s H(s)<s^{2} H(s)=s^{2}|\phi(s)|^{2}$; so that the map $t \rightarrow t|\phi(t)|$ is also a strictly increasing function. We conclude that the image of the closed unit interval under $t \phi$ is a Jordan arc $\Gamma$. Since $z^{k} f=|z|^{2 k} \phi\left(|z|^{2 k}\right)$ the image of $D$ under $z^{k} f$ is exactly $\Gamma$ and distinct circles about 0 are mapped into distinct points of $\Gamma$.

Proof of Theorem II. Let $\Gamma$ be as in Lemma 3.3. Since $\Gamma$ is a Jordan arc, it has connected complement and no interior. By Theorem 3.1, $P(\Gamma)=C(\Gamma)$. Let $h$ be the inverse of the map $t \rightarrow t \phi(t)$ which traced out $\Gamma$ and let $g=h \circ\left(z^{k} f\right)$. Since $h \in C(\Gamma), h \in P(\Gamma)$ and so $g \in P_{f}$. Further, the level sets of $g$ are precisely the circles in $D$ with center at 0 and the restriction of $P_{f}$ to any circle about the origin is dense in all continuous functions on that circle $\left(P_{f}\right.$ restricted to the circle contains $z$ and $\bar{z}^{k}$, hence, $z$ and $\bar{z}$ ). We conclude from Theorem 3.2 that $P_{f}=C(D)$.

\section{REFERENCES}

1. A. Browder, Introduction to function algebras, Benjamin, New York, 1969.

2. L. De Branges, The Stone-Weierstrass theorem, Proc. Amer. Math. Soc. 10 (1959), 822-824.

3. K. Preskenis, Approximation on disks, Trans. Amer. Math. Soc. 171 (1972), 445-467. 
4. __ Another view of the Weierstrass theorem, Proc. Amer. Math. Soc. S4 (1976), 109-113.

5. J. Väisälä, Lectures on n-dimensional quasi-conformal mappings, Lecture Notes in Math., vol. 229, Springer-Verlag, Berlin and New York, 1971.

6. J. Wermer, Approximation on a disk, Math. Ann. 155 (1965), 331-333.

7. __ Polynomially convex disks, Math. Ann. 158 (1965), 6-10.

Departmant of Mathematics, Boston College, Chestnut Hill, Massachusetts 02167

Current address: Department of Mathematics, Framingham State College, Framingham Center, Massachusetts 01701 